\title{
Intrabronchial Route of Administration
}

National Cancer Institute

\section{Source}

National Cancer Institute. Intrabronchial Route of Administration. NCI Thesaurus. Code C38225.

Administration of a drug within a bronchus or bronchi. The intrabronchial drug delivery can be used for both local and systemic drug action. 